Supporting information for the manuscript

\title{
Restoring Resolution in Biological Solid-State NMR under Conditions of off-Magic-Angle Spinning
}

Riddhiman Sarkar, ${ }^{a, b}$ Diana C. Rodriguez Camargo ${ }^{a, b}$, Guido Pintacuda ${ }^{c}$ and Bernd Reif ${ }^{a, b *}$

a Helmholtz-Zentrum München (HMGU), Deutsches Forschungszentrum für Gesundheit und Umwelt, Ingolstädter Landstr. 1, 85764 Neuherberg, Germany

b Munich Center for Integrated Protein Science (CIPS-M) at Department Chemie, Technische Universität München (TUM), Lichtenbergstr. 4, 85747 Garching, Germany

c Université de Lyon, Institut de Sciences Analytiques, Centre de RMN à Très Hauts Champs, 5 rue de la Doua, 69100 Villeurbanne, France 


\section{Experimental}

The perdeuterated, microcrystalline $\mathrm{SH} 3$ sample was prepared as described previously ${ }^{1}$. S3E and HSQC experiments were recorded without the aid of gradients for coherence order selection and water suppression. The experimental implementation of S3E and HSQC experiments is schematically represented in Figure S1. In all experiments, a recycle delay of $1.5 \mathrm{~s}$ was employed. The number of points in the indirect dimension was set to 326 yielding a $t_{1}{ }^{\max }$ of $46 \mathrm{~ms} .8$ and 128 scans were accumulated for each FID for the HSQC and the S3E experiments, respectively. In the HSQC experiments, WALTZ-16 decoupling was used during acquisition using a proton $\mathrm{RF}$ field strength of $2 \mathrm{kHz}$. The total experimental time for both the experiments amounted to $9 \mathrm{~h} 5 \mathrm{~min}$. The experiments were performed at a magnetic field strength of $23.5 \mathrm{~T}$, corresponding to a proton Larmor frequency of $1 \mathrm{GHz}$. The MAS rotation frequency was adjusted to $60 \mathrm{kHz}$. The temperature of the cooling gas was set to $33^{\circ} \mathrm{C}$. The effective temperature of the sample was on the order of $+10^{\circ} \mathrm{C}$ as found by the temperature dependent water chemical shift.
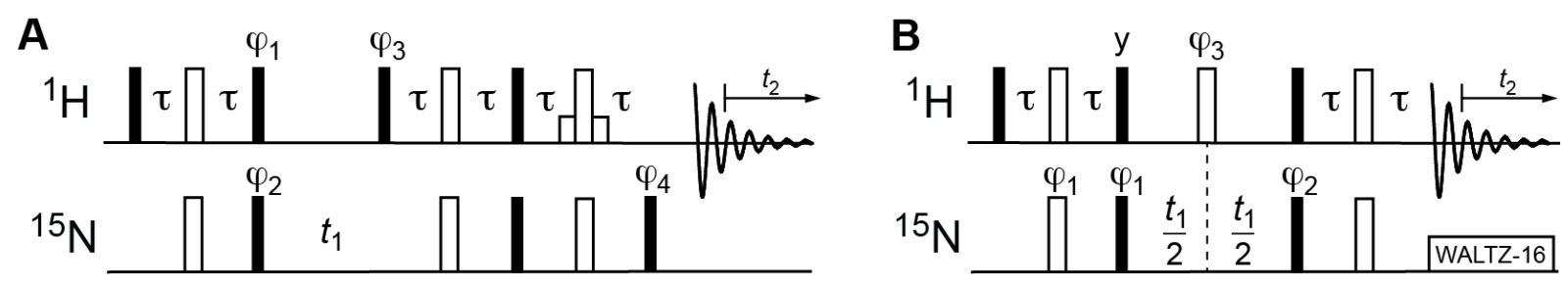

Figure S1: S3E (A) and HSQC (B) pulse sequences to record ${ }^{1} \mathrm{H},{ }^{15} \mathrm{~N}$ correlations. ${ }^{2}$ Filled and open rectangles denote $90^{\circ}$ and $180^{\circ}$ pulses, respectively. In the experiments $\tau$ was set to $2.3 \mathrm{~ms}$. Lower, open bars in $(A)$ denote selective pulses used for water suppression. The following phase cycles are used: $(A) \Phi_{1}=-y ; \Phi_{2}=y,-y,-x, x ; \Phi_{3}=y,-y ; \Phi_{4}=x,-x ; \Phi_{R}=x,-x,-y$, $y,-x, x,-y, y .(B)$ the following phase cycles are used: $\Phi_{1}=x,-x ; \Phi_{2}=2(x), 2(-x) ; \Phi_{3}=4(y), 4(-$ $y) ; \Phi_{R}=x,-x,-x, x$. If not otherwise indicated, all other pulse phases are set to $x$. 


\section{Theory}

The coherent Hamiltonian active during the evolution in the indirect dimension of the S3E experiment is given as

$$
\begin{aligned}
H^{i s o} & =\delta_{N}^{i s o} N_{z}+2 \pi J N_{z} H_{z} \\
H^{\text {aniso }} & =\delta_{N}^{\text {aniso }} N_{z}+2 d N_{z} H_{z}
\end{aligned}
$$

Here, $\delta_{\mathrm{N}}{ }^{\text {iso }}, \delta_{\mathrm{N}}^{\text {aniso }}, J$ and $d$ are the isotropic, anisotropic chemical shifts of ${ }^{15} \mathrm{~N}$, the scalar and the dipolar ${ }^{1} \mathrm{H},{ }^{15} \mathrm{~N}$ coupling constants, respectively. At the magic-angle, the anisotropic part of the Hamiltonian vanishes, since $d^{2}{ }_{00}\left(\tan ^{-1} \sqrt{2}\right)=0$, where $d^{2}{ }_{00}(\beta)$ corresponds to the reduced Wigner rotation matrix element, and $\beta$ to the rotor axis. When the rotor axis is not set to the magic angle, a residual anisotropy is retained. Following the notation of Pileio et al., ${ }^{3}$ we rewrite

$$
d_{00}^{2}\left(\tan ^{-1} \sqrt{2}+\Delta \vartheta_{R L}\right) \approx \sqrt{2} \Delta \vartheta_{R L}
$$

Assuming that the spinning angle is mis-set by $\Delta \vartheta_{\mathrm{RL}}$ with respect to the magic angle, the anisotropic part of the Hamiltonian becomes

$$
H^{\text {aniso }}=\sqrt{2} \Delta \vartheta_{R L}\left(\delta_{N}^{\text {aniso }} N_{z}+2 d N_{z} H_{z}\right) d_{00}^{2}\left(\beta_{P R}\right)
$$

Where $\beta_{\mathrm{PR}}$ denotes the angle between the principal axis of the molecule fixed frame and the rotor frame. Using the definition for $H^{a}$ and $H^{\beta}$, we obtain

$$
\begin{aligned}
H^{\text {aniso }} & =d_{00}^{2}\left(\beta_{P R}\right)\left(\sqrt{2} \Delta \vartheta_{R L}\right)\left[\delta_{N}^{\text {aniso }}+d\left(H^{\alpha}-H^{\beta}\right)\right] N_{z} \\
& =d_{00}^{2}\left(\beta_{P R}\right)\left(\sqrt{2} \Delta \vartheta_{R L}\right)\left[\left(\delta_{N}^{\text {aniso }}+d\right) N_{z} H^{\alpha}+\left(\delta_{N}^{\text {aniso }}-d\right) N_{z} H^{\beta}\right]
\end{aligned}
$$

The isotropic part of the Hamiltonian adopts the form

$$
H^{i s o}=\left[\left(\delta_{N}^{i s o}+\pi J\right) N_{z} H^{\alpha}+\left(\delta_{N}^{i s o}-\pi J\right) N_{z} H^{\beta}\right]
$$

In the indirect dimension, the density operator $\rho=H^{\beta} \mathrm{N}^{-}$evolves with the frequency $\left(\delta_{\mathrm{N}}{ }^{\text {iso }}\right.$ $\Pi J)$, while the shape of the peak is governed by the residual anisotropy $d^{2}{ }_{00}\left(\beta_{\mathrm{PR}}\right)\left(\sqrt{2} 2 \Delta \vartheta_{\mathrm{RL}}\right)\left(\delta_{\mathrm{N}}{ }^{\text {aniso }}-d\right)$. Similarly, $\rho=H^{\mathrm{a}} \mathrm{N}^{-}$evolves with the frequency $\left(\delta_{\mathrm{N}}{ }^{\text {iso }}+\pi J\right)$, while the peak shape is governed by the sum of the anisotropy $d^{2}{ }_{00}\left(\beta_{\mathrm{PR}}\right)\left(\sqrt{ } 2 \Delta \vartheta_{\mathrm{RL}}\right)\left(\delta_{\mathrm{N}}{ }^{\text {aniso }}+d\right)$ under the influence of the anisotropy. To explicitly calculate the spectrum, powder averaging over all crystallite orientations $\beta_{\mathrm{PR}}$ has to be taken into account. 


\section{References}

(1) Chevelkov, V.; Rehbein, K.; Diehl, A.; Reif, B. Ultrahigh resolution in proton solidstate NMR spectroscopy at high levels of deuteration. Angew Chem Int Edit 2006, 45, 3878-3881.

(2) Rance, M.; Loria, J. P.; Palmer, A. G. Sensitivity improvement of transverse relaxation-optimized spectroscopy. J Magn Reson 1999, 136, 92-101.

(3) Pileio, G.; Guo, Y.; Pham, T. N.; Griffin, J. M.; Levitt, M. H.; Brown, S. P. Residual dipolar couplings by off-magic-angle spinning in solid-state nuclear magnetic resonance Spectroscopy. J Am Chem Soc 2007, 129, 10972-+. 